\title{
Signalparameter zur Ortung von HPEM-Quellen
}

\author{
R. Kanyou Nana, W. Kranzpiller, N. Sonnenberg, and R. Nguete Tsamo \\ Thales Deutschland, Defence \& Security Systems \\ Correspondence to: R. Kanyou Nana (richard.kanyou@ thalesgroup.com)
}

Zusammenfassung. In diesem Beitrag wird anhand von Signalproben untersucht, mit welchen geeigneten Signalparametern die Ortung von aktiven leistungsstarken elektromagnetischen Quellen (sog. HPEM-Quellen) optimal durchgeführt werden kann. Da sich die Positionsberechnung durch die Verarbeitung von Laufzeitdifferenzen ergibt, beschränkt sich die Untersuchung auf die Bestimmung der Laufzeitdifferenzen. Betrachtet werden sowohl Signalausschnitte definierter Länge sowie eindeutig identifizierbare Ereigniszeitpunkte. Im ersten Fall werden aus der Korrelation der Signalausschnitte im Frequenzbereich die Laufzeitdifferenzen bestimmt. Hierbei wird durch Variation der Anzahl der Abtastwerte eines Signalausschnitts die erforderliche Signaldauer ermittelt. Im zweiten Fall werden zuerst aus den direkt erfassten Signaldaten die Einhüllenden gewonnen. Bezüglich eines vordefinierten Referenzwertes werden diese Einhüllenden dann korrigiert. Mit Hilfe eines festgelegten Schwellwertes wird abschließend aus den korrigierten Hüllkurven die Differenz der Ereigniszeitpunkte gebildet und somit die Laufzeitdifferenz im Zeitbereich bestimmt.

\section{Einleitung}

In der modernen Luftverkehrstechnik werden zunehmend elektronische Systeme eingesetzt, um eine zuverlässige und reibungslose Abwicklung des Betriebs sicherzustellen. In einigen Fällen genügt alleine eine Fehlfunktion wie z.B. die fehlerhafte Datenübertragung oder der Ausfall einer Systemkomponente, um erhebliche Störungen im Betriebsablauf des Systems zu verursachen. Fehlfunktionen können beispielsweise durch Felder von HPEM-Quellen hervorgerufen werden. Angesichts der steigenden Verbreitung und Verfügbarkeit von HPEM-Wirkmitteln sowie der beobachtbaren Abnahme der Störfestigkeit moderner elektronischer Systeme gegenüber EM-Feldern ist es notwendig, ein Schutzkonzept zu entwicklen, welches die Detektion und Lokalisie- rung von HPEM-Angriffen zuverlässig, zeitnah und störsicher ermöglicht. Das hier vorgestellte Ortungsverfahren basiert auf der Vermessung der Signallaufzeitdifferenzen vom Emitter zu den Empfängern (sog. Time Difference of Arrival, TDoA-Verfahren), wobei eine zeit- und frequenzsynchrone Signaldatenerfassung vorauszusetzen ist.

Der vorliegende Bericht ist neben der Einleitung in drei weitere Abschnitte unterteilt. In Kapitel 2 werden die erforderlichen Grundlagen zusammengestellt, auf denen die folgenden Aufführungen aufbauen. Dazu gehört zum Teil die Erläuterung des TDoA-Verfahrens. Hier wird das Prinzip der Hyperbelortung in 2-D vorgestellt. Des Weiteren werden in diesem Kapitel die typischen Pulse von HPEM-Quellen sowie ihre wichtigsten Parameter zur Laufzeitberechnung präsentiert. Das Kapitel schließt mit der Darstellung des bei den numerischen Berechnungen verwendeten Modells der Empfangsstationen ab. Im Anschluss an die Grundlagen werden in Kapitel 3 zwei Verfahren vorgestellt, welche die optimale Bestimmung der Laufzeitdifferenzen gewährleisten. Anhand von Signalproben werden hierbei die Zeitverzögerungen zwischen den Empfangssignalen sowie die räumliche Trennung der Empfangsstationen - hier modelliert durch unterschiedlich gewichtete Signalamplituden künstlich erzeugt. Des Weiteren wird in diesem Kapitel ein Vergleich beider Methoden bezüglich der erzielbaren Genauigkeit und abhängig von eingesetzter HPEM-Pulsform durchgeführt. Dadurch wird festgestellt, welche Methode für welche HPEM-Pulsform am geeignetsten ist. Der Bericht schließt mit einer Zusammenfassung in Kapitel 4 ab.

\section{Theoretische Grundlagen}

\subsection{TDoA-Verfahren in 2-D}

Punkte, deren Entfernungsdifferenz zu zwei gegebenen festen Punkten konstant ist, liegen auf einer Hyperbel 
Tabelle 1. Aus Abbildungen 2-4 gewonnene Signalparameter für die TDoA-Berechnung.

\begin{tabular}{llll}
\hline Parameter & HPM (SUPRA) & DS (vom Typ 110T) & UWB (PBG5 \& kleines Horn) \\
\hline Spitzenwert (E-Feld) & ca. $50 \mathrm{kV} \mathrm{m}^{-1}$ in $15 \mathrm{~m}$ & ca. $230 \mathrm{kV} \mathrm{m}^{-1}$ in $1 \mathrm{~m}$ & ca. $0.78 \mathrm{kV} \mathrm{m}^{-1}$ in $10 \mathrm{~m}$ \\
Anstiegszeit & ca. $142 \mathrm{~ns}$ & ca. $2 \mathrm{~ns}$ & ca. $420 \mathrm{ps}$ \\
Pulsdauer & ca. $550 \mathrm{~ns}$ & ca. $20 \mathrm{~ns}$ & ca. $2 \mathrm{~ns}$ \\
Bandbreite & ca. $5 \mathrm{MHz}$ & ca. $200 \mathrm{MHz}$ & ca. $2 \mathrm{GHz}$ \\
\hline
\end{tabular}

Tabelle 2. Vergleich der TDoA-Werte von korrigierten und unkorrigierten Signaldaten bei der Verwendung von HPM-Pulsen.

\begin{tabular}{llll}
\hline \multirow{2}{*}{ Delay } & $10 \%-\mathrm{TDoA}: \mathrm{U}_{\mathrm{HF}}(\mathrm{t}) / \mathrm{U}_{\mathrm{HF}}(\mathrm{t})_{\mid \text {kor }}$ & $10 \%$-TDoA: $\mathrm{U}_{\mathrm{BP}}(\mathrm{t}) / \mathrm{U}_{\mathrm{BP}}(\mathrm{t})_{\mid \text {kor }}$ & $10 \%-\mathrm{TDoA}: \mathrm{U}(\mathrm{k} \Delta \mathrm{t}) / \mathrm{U}(\mathrm{k} \Delta \mathrm{t})_{\mid \text {kor }}$ \\
& $50 \%-\mathrm{TDoA}: \mathrm{U}_{\mathrm{HF}}(\mathrm{t}) / \mathrm{U}_{\mathrm{HF}}(\mathrm{t})_{\mid \text {kor }}$ & $50 \%$-TDoA: $\mathrm{U}_{\mathrm{BP}}(\mathrm{t}) / \mathrm{U}_{\mathrm{BP}}(\mathrm{t})_{\mid \text {kor }}$ & $50 \%$-TDoA: $\mathrm{U}(\mathrm{k} \Delta \mathrm{t}) / \mathrm{U}(\mathrm{k} \Delta \mathrm{t})_{\mid \mathrm{kor}}$ \\
\hline \multirow{2}{*}{$80 \mathrm{~ns}$} & $91.26 \mathrm{~ns} / 80 \mathrm{~ns}$ & $93.18 \mathrm{~ns} / 80.98 \mathrm{~ns}$ & $98.09 \mathrm{~ns} / 85.04 \mathrm{~ns}$ \\
& $240.75 \mathrm{~ns} / 80 \mathrm{~ns}$ & $180.64 \mathrm{~ns} / 80.58 \mathrm{~ns}$ & $190.09 \mathrm{~ns} / 84.72 \mathrm{~ns}$ \\
$60 \mathrm{~ns}$ & $71.27 \mathrm{~ns} / 60 \mathrm{~ns}$ & $73.6 \mathrm{~ns} / 59.82 \mathrm{~ns}$ & $77.46 \mathrm{~ns} / 63 \mathrm{~ns}$ \\
& $220.78 \mathrm{~ns} / 60 \mathrm{~ns}$ & $161.42 \mathrm{~ns} / 60.48 \mathrm{~ns}$ & $180 \mathrm{~ns} / 63.57 \mathrm{~ns}$ \\
$40 \mathrm{~ns}$ & $51.29 \mathrm{~ns} / 40 \mathrm{~ns}$ & $54.18 \mathrm{~ns} / 40.61 \mathrm{~ns}$ & $57.05 \mathrm{~ns} / 42.8 \mathrm{~ns}$ \\
& $200.81 \mathrm{~ns} / 40 \mathrm{~ns}$ & $143.04 \mathrm{~ns} / 40.17 \mathrm{~ns}$ & $150.39 \mathrm{~ns} / 42.22 \mathrm{~ns}$
\end{tabular}

(Bronstein et al., 1999). Für eine beliebige sich auf der Hyperbel befindliche EM-Quelle E, gilt die folgende Beziehung zwischen Abstand-Unterschied und Laufzeitdifferenz zweier Signale

$d_{\mathrm{ES}_{1}}-d_{\mathrm{ES}_{2}}=c \cdot \underbrace{\left(t_{\mathrm{ES}_{1}}-t_{\mathrm{ES}_{1}}\right)}_{\Delta \tau_{1}}=c \cdot \Delta \tau_{1}$

wobei $S_{1}$ und $S_{2}$ die festgelegten Punkte repräsentieren. $\mathrm{Zu}$ jedem ermittelten Wert der Signallaufzeitdifferenz zweier Empfangsstationen gehört also eine Hyperbel, welche durch die gesuchte Position des Emitters verläuft. Um die Emitterposition als Schnittpunkt zweier Hyperbeln bestimmen zu können, ist ein dritter Empfänger zur Ableitung der zweiten Hyperbel erforderlich. Die sog. Hyperbelortung basiert somit auf der Vermessung der Signallaufzeitdifferenzen vom Sender zu den Empfängern wie Abb. 1 zeigt.

\subsection{Pulse von HPEM-Quellen und ihre technischen Parameter}

\subsubsection{Pulse von HPEM-Quellen}

Im Begriff HPEM-Quellen werden unterschiedliche energiereiche Wirkmittel zusammengefasst, die transiente EMFelder mit hohen Amplituden und schnellen Anstiegszeiten emittieren können. Eine ausführliche Beschreibung derartiger Quellen ist z.B. in Giri (2004), Taylor und Giri (1994) und Schmidt (2002) zu finden. Typische Pulsformen von HPEM-Quellen sind HPM (High Power Microwave), DS (Damped Sinusoidal) und UWB (Ultra-Wide-Band).

HPM-Pulse werden durch ihre schmalbandigen und sehr leistungsstarken Signalformen charakterisiert. Die Gesamtenergie ist in einem sehr schmalen Frequenzbereich um die Mittenfrequenz konzentriert. Abb. 2 stellt die Zeit- und Frequenzverläufe eines einzelnen HPM-Pulses dar, welcher in der Absorberhalle des Wehrwissenschaftlichen Instituts für Schutztechnologien (WIS) in Munster mit dem HPMSimulator SUPRA erzeugt und mit einem D-Dot Freifeldsensor (als induzierte Spannung) aufgenommen wurde.

DS-Pulse zeichnen sich durch ihre breitbandigen und leistungsstarken Signalformen aus. Der Hauptteil der Gesamtenergie ist in einem breiten Frequenzbereich um die Mittenfrequenz konzentriert. In Abb. 3 sind ebenfalls die Zeit- und Frequenzverläufe eines derartigen Einzelpulses zu sehen. Das Signal stammt von einer DS-Quelle (vom Typ 110T) des Herstellers Diehl. Mit einem breitbandigen D-Dot Freifeldsensor wurde die durch das EM-Feld induzierte Spannung vermessen. Wegen der breitbandigen Eigenschaft des Messsignals resultiert die Integration dieser Spannung - unter Berücksichtigung der äquivalenten Antennenfläche - zu dem dargestellten elektrischen Feld.

UWB-Pulse weisen eine extrem breitbandige und ebenfalls leistungsstarke Signalform auf. Da aber die Pulsenergie über einen sehr breiten Frequenzbereich verteilt ist, besitzen die einzelnen Spektralanteile nur einen geringen Bruchteil der Gesamtenergie wie dies in Abb. 4 zu sehen ist.

Die oben genannten HPEM-Pulse können auch als Impulspakete (sog. Bursts) auftreten. Zur Ermittlung der Signallaufzeitdifferenzen werden deswegen auch HPEM-Bursts betrachtet. Diese sind insbesondere durch die Wiederholrate der einzelnen Pulse in einem Burstpaket als auch durch die Burstdauer sowie die Burstperiode bestimmt. Zur Verdeutlichung stellt Abb. 5 die Definition der wichtigen Kenngrößen eines idealen UWB-Bursts dar. 
Tabelle 3. Zusammenstellung der TDoA-Ergebnisse von verschiedenen HPEM-Pulsformen zwecks Vergleich beider Verfahren.

\begin{tabular}{llllllll}
\hline Delay [ns]: & 80 & 70 & 65 & 60 & 56.5 & 40 & 20 \\
\hline $\begin{array}{l}\text { TDoA [ns] HPM: } \\
\text { 50\%-Max/Korrelation }\end{array}$ & $84.72 / 84$ & $74.4 / 72$ & $69 / 68$ & $63.57 / 64$ & $59.59 / 60$ & $42.22 / 44$ & $21.75 / 20$ \\
$\begin{array}{l}\text { TDoA [ns] DS: } \\
\text { 50\%-Max/Korrelation }\end{array}$ & $78.66 / 80$ & $70.36 / 72$ & $65.59 / 64$ & $59.7 / 60$ & $56.5 / 56$ & $43.12 / 40$ & $19.72 / 20$ \\
$\begin{array}{l}\text { TDoA [ns] UWB: } \\
\text { 50\%-Max/Korrelation }\end{array}$ & $41.66 / 80$ & $28.5 / 72$ & $24.55 / 64$ & $27.27 / 60$ & $25.5 / 56$ & $21.14 / 40$ & $23.49 / 20$ \\
\hline
\end{tabular}

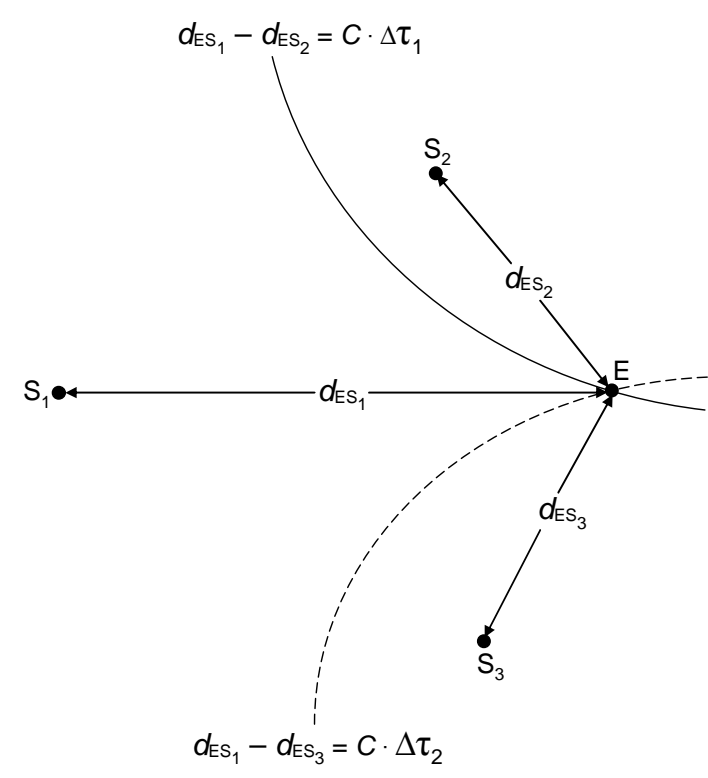

Abb. 1. Erläuterung des TDoA-Prinzipes.

\subsubsection{Technische Parameter von HPEM-Pulsen}

In Bezug auf die Berechnung der Laufzeitdifferenzen sind zur Charakterisierung von HPEM-Pulsen folgende Signalparameter hilfreich:

- Spitzenwert: Signalamplitude, bei der das Maximum erreicht ist. Diese Kenngröße, die sehr stark von den Eigenschaften des Übertragungskanals (Freiraumdämpfung, Mehrwegeausbreitung etc.) abhängt, wird hier benutzt, um Ereigniszeitpunkte bei der Zeitbereichsanalyse abzuleiten.

- Anstiegszeit: Dauer, innerhalb der das Signal von $10 \%$ auf $90 \%$ des Spitzenwertes gestiegen ist. Sie ist verantwortlich für das Auftreten hochfrequenter Anteile im Signalspektrum.

- Pulsdauer: zeitlicher Abstand zwischen Anfang und Ende eines Einzelpulses. Diese Größe hängt stark von dem eingesetzten Impuls ab und wird zur Ermittlung der Signalblocklänge ausgenutzt, welche zur Durchführung der Korrelation benötigt wird.
- Bandbreite: ursprünglich definiert als Differenz zwischen oberer und unterer Grenzfrequenz. Bei ausgewähltem AD-Wandler (hier $80 \mathrm{MHz}$ Erfassungsbereich) bestimmt die Signalbandbreite die Form des Ausgangssignals des HF-Frontends. Wie mittels Simulation festgestellt wurde, resultiert aus der Verwendung von HPM-Pulsen die Einhüllende des HF-Pulses als Ausgangssignal. Bei DS- und UWB-Pulsen dagegen ergibt sich wegen der Breitbandigkeit näherungsweise die Impulsantwort des schmalbandigeren Filters.

Einen Eindruck von der Größenordnung der eben definierten Signalparameter liefert Tabelle 1. Zur Ermittlung dieser wichtigen Kenngrößen wurden die HPEM-Pulsformen aus den Abbildungen 2-4 verwendet.

\subsection{Modell einer Empfangsstation}

Das in Abb. 6 skizzierte Blockschaltbild repräsentiert das vereinfachte Modell eines Ortungssensors. Durch Abmischung des an einer Empfangsstation befindlichen HFSignals ins Basisband wird das äquivalente Bandpaßsignal mittels ZF-Filterung des gemischten Signals gewonnen. In Bezug auf die realen Sensoren werden diese Operationen von dem Analog-Modul durchgeführt. Das gefilterte niederfrequente Signal wird dann mit einem $(250 \mathrm{MHz})$ ADWandler abgetastet. Unter Einsatzbedingungen übernimmt der Digitalteil des HF-Frontends diese Teilaufgabe. Erst nach diesem Schritt findet im Steuerrechner zunächst die HPEM-Signaldetektion und anschließend die Eliminierung bzw. Reduktion der Signalreflexionen statt. Die Berücksichtigung der Signalreflexionen ist wegen der im Einsatzgebiet befindlichen Hindernisse notwendig. Dies führt zu einem möglichst sauberen Signal in Hinsicht auf die geforderte LoS-Bedingung (Line of Sight). Die Signaldaten werden abschließend zum OrtungsController übertragen, der die Berechnung sämtlicher Laufzeitdifferenzen durchführt.

\section{Laufzeitberechnungsverfahren}

Die beiden in diesem Kapitel vorgestellten Verfahren zur Bestimmung der Signallaufzeitdifferenzen setzen voraus, dass sowohl die Signalerfassung als auch die HPEM-Detektion 

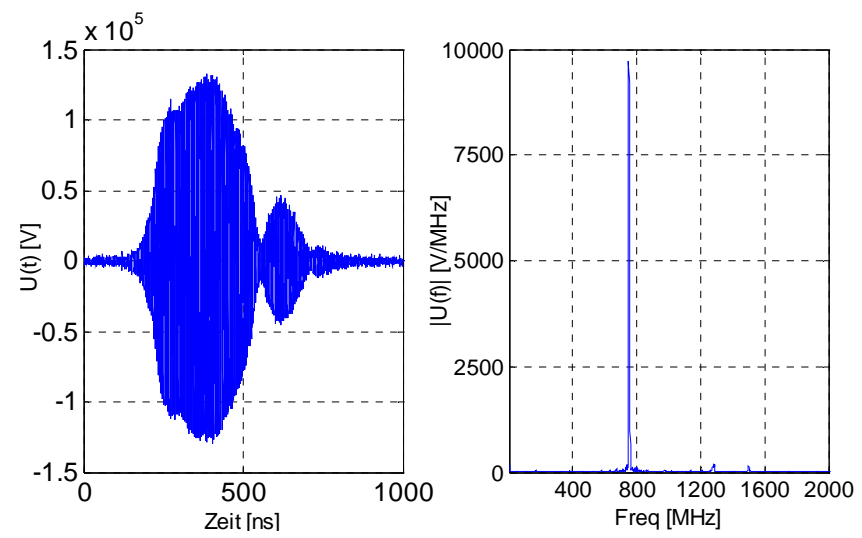

Abb. 2. Einmalig auftretender HPM-Puls im Zeit- und Frequenzbereich.
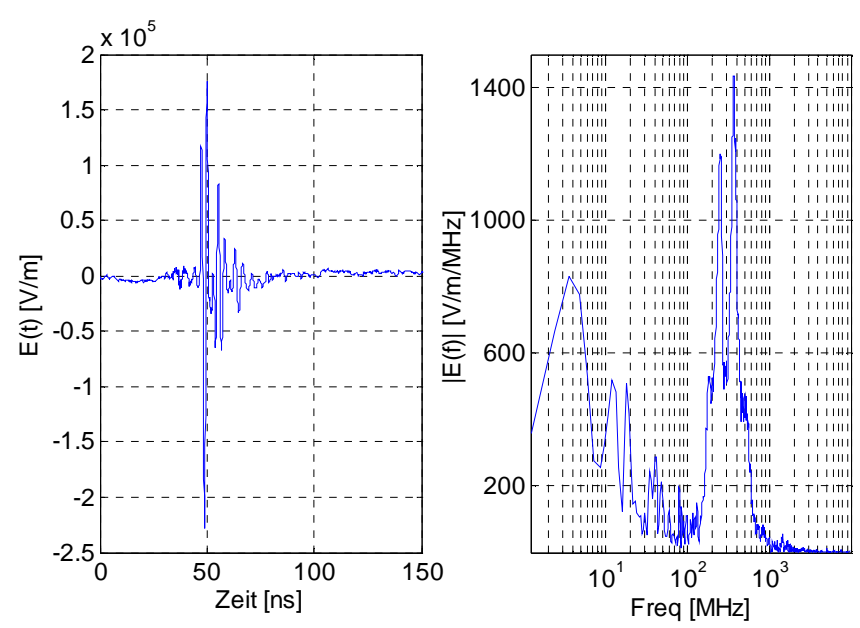

Abb. 3. Zeit- und Frequenzverläufe eines einmalig auftretenden elektrischen DS-Pulses: $1 \mathrm{~m}$ Entfernung \& horizontale Ausrichtung.

sowie das Extrahieren des LoS-Signals aus dem Empfangssignal bereits stattgefunden haben. Für Simulationszwecke wird also lediglich das Signal vom direkten Weg betrachtet. Die Zeitverzögerung wird künstlich erzeugt. Die räumliche Trennung der Sensosen wird durch unterschiedlich gewichtete Signalamplituden in Betracht genommen, wobei die Form der einzelnen Empfangssignale gleich anzunehmen ist. Abb. 7 stellt exemplarisch die Zeit- und Frequenzverläufe des obigen HPM-Einzelpulses bei den einzelnen Verarbeitungsstufen des HF-Frontends dar; die Frequenz des Lokaloszillators beträgt $690 \mathrm{MHz}$.

Hier ist die Transformation des HF-Signals in NF-Signal deutlich zu erkennen. Es lässt sich außerdem die Bandbreitenreduktion durch Abtastung des erhaltenen NF-Signals beobachten. Auf diese Art und Weise werden im Folgenden alle HPEM-Signale numerisch verarbeitet.
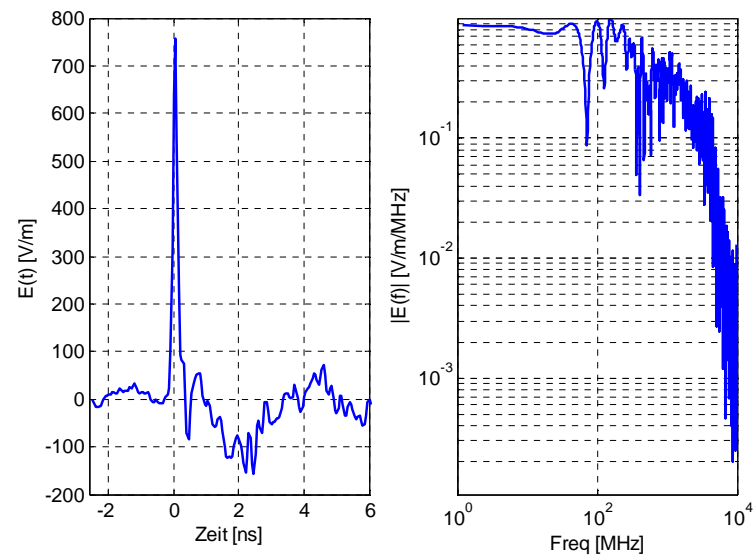

Abb. 4. Zeit- und Frequenzverläufe eines einzelnen UWB-Pulses des Systems bestehend aus einem PBG5-Generator und einer kleinen Hornantenne: $10 \mathrm{~m}$ Entfernung.

\subsection{Zeitbereichsanalyse}

Bei diesem Verfahren handelt es sich um eine direkte Ermittlung der Laufzeitdifferenzen durch Differenzbildung der Ereigniszeitpunkte. Da hier die Festlegung eines definierten Identifizierers (Schwellendetektor, der den Ereigniszeitpunkt bei überschreiten einer bestimmten Amplitudenschwelle bestimmt) vorauszusetzen ist, wird der Fokus auf den $10 \%$ , $50 \%$ - bzw. $90 \%$-Wert der Maximalamplitude gelegt. Ohne Korrekturmaßnahmen lässt sich intuitiv die Laufzeitdifferenz wie folgt ermitteln: Zunächst werden die Hüllkurven bestimmt. Dann wird ein Schwellwert festgelegt. Die Differenzbildung der Ereigniszeitpunkte ergibt schließlich die gesuchte Laufzeitdifferenz. Am Beispiel der HPM-Pulsform stellt Abb. 8 die Signale von zwei räumlich verteilten Empfangsstationen sowie die zugehörigen Hüllkurven dar.

Es ist offensichtlich, dass sich die TDoA-Ergebnisse bei $10 \%$ - und $50 \%$-Max stark voneinander unterscheiden. Die Gültigkeit der Zeitbereichsanalyse verlangt also eine Korrektur der Signaldaten. Die hier angewandte Korrekturmethode ähnelt der Funktionsweise von Systemen, die mit einem AGC (Automatic Gain Control) ausgestattet sind. Es geht darum, den Pegel ankommender Nutzsignale auf einen bestimmten Referenzwert zu normalisieren, wobei als Nutzsignal die Hüllkurve des HPEM-Pulses gemeint ist. Mathematisch ausgedrückt, handelt es sich um die Bestimmung des Skalierungsfaktors $K$ bei der folgenden Gleichung, wobei $x(n)$ und $y(n)$ jeweils den Abtastwert des Eingangs- und Ausgangssignals darstellen.

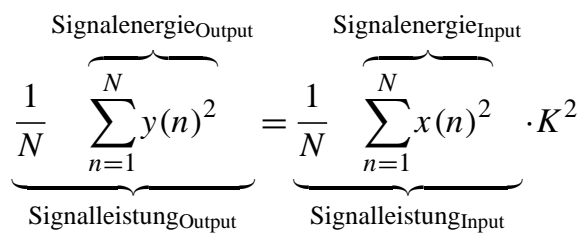




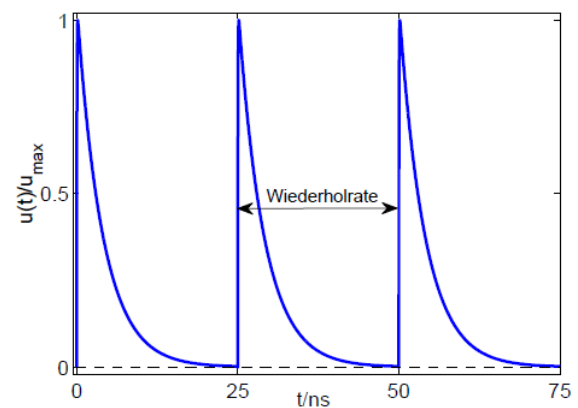

Abb. 5. Zur Definition der Kenngrößen eines HPEM-Impulspakets.

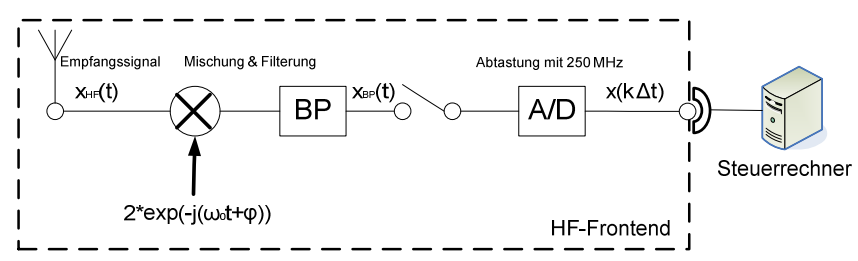

Abb. 6. Vereinfachte Darstellung einer Empfangsstation.

$K$ sorgt dafür, dass unabhängig von der Eingangsleistung stets die gleiche Ausgangsleistung bereitgestellt wird. Mit der vereinfachten Darstellung von Gl. (2) in Gl. (3) kann $K$ auch als Größe angesehen werden, die mit den Abtastwerten beliebiges Eingangssignals zu multiplizieren ist, damit der Erhalt einer einheitlichen Energie gewährleistet ist.

$\sum_{n=1}^{N} y(n)^{2}=\sum_{n=1}^{N}[x(n) \cdot K]^{2}$

Durch Umstellung von Gl. (2) nach $K$ ergibt sich nach einigen Vereinfachungen die folgende Beziehung zwischen dem Skalierungsfaktor $K$, der Energie des Eingangssignals $E_{\text {in }}$, der vordefinierten Ausgangsleistung $P_{\text {out }}$ und der Anzahl der Samples $N$.

$K=\sqrt{\frac{\frac{1}{N} \sum_{n=1}^{N} y(n)^{2} \cdot N}{\sum_{n=1}^{N} x(n)^{2}}}=\sqrt{\frac{P_{\mathrm{out}} \cdot N}{E_{\mathrm{in}}}}$

Eine gemeinsame Darstellung der unkorrigierten und korrigierten Einhüllenden der oben beschriebenen HPM-Pulse findet sich in Abb. 9.

Ein Vergleich der TDoA-Ergebnisse, die mit Hilfe von korrigierten und unkorrigierten Hüllkurven gewonnen wurden, ist in Tabelle 2 für verschiedene Zeitverzögerungen zu sehen. Im Gegensatz zu unkorrigierten Daten lassen sich mit korrigierten Daten sehr gute Ergebnisse erzielen. Der hier zu beobachtende kleine Unterschied zwischen Ist- und Soll-TDoA ist auf die Verarbeitungskette des HF-Frontends

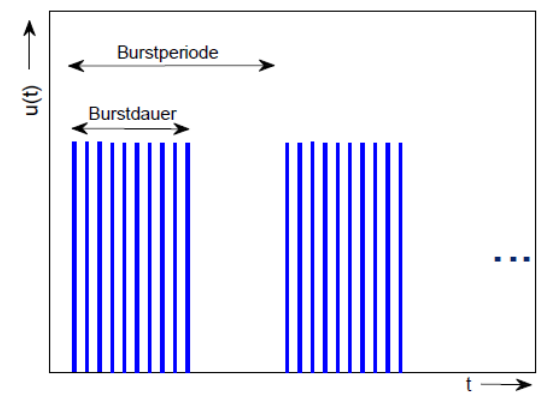

zurückzuführen. Unter Verwendung von DS- und UWBPulsformen zeigte das Verfahren weiterhin seine Brauchbarkeit. Bei UWB-Pulsen war allerdings der Einfluss der Verarbeitungskette noch ausgeprägter.

\subsection{Frequenzbereichsanalyse}

Bei diesem Verfahren handelt es sich um die Bestimmung der Signallaufzeitdifferenzen mittels Korrelation von Signalausschnitten im Frequenzbereich. Hier wird zuerst eine Fensterfunktion ausgewählt, mit der die Eingangssignale multipliziert werden, um Signal-Unstetigkeit am linken und rechten Ende der Datenblöcke zu vermeiden. Danach findet die FFT (Fast Fourier Transformation) der erhaltenen Signale statt. Es folgt anschließend für jedes Sensorpaar die Multiplikation des Signals 1 mit dem konjugiert komplexen Signal 2. Nach dieser Operation wird die Kreuzkorrelation durch Bildung der IFFT (Inverse FFT) des Zwischenergebnisses gewonnen. Aus der Position des Maximums des Betrages der Kreuzkorrelation erhält man schließlich den gesuchten TDoA-Wert. Am Beispiel eines HPM-Bursts - bestehend aus einer 4Impuls Sequenz - stellt Abb. 10 die Segmentierung seiner abgetasteten NF-Teilsignale $\mathrm{U}(\mathrm{k} \Delta \mathrm{t})$ in Blöcken von 256 Samples sowie die daraus resultierenden TDoA-Ergebnisse dar. Die künstlich generierte Zeitverzögerung beträgt $60 \mathrm{~ns}$.

Mit dem TDoA-Ergebnis des kompletten Bursts als Bezugsgröße, ist in Abb. 10 deutlich zu erkennen, dass eine Übereinstimmung der TDoA-Werte nur zu gewährleiten ist, falls der Signalblock mindestens einen kompletten Einzelpuls in seinem Zeitfenster enthält. Diese Feststellung wurde auch bei DS- und UWB-Bursts gemacht, wie Abbildungen 11-12 im Falle einer 8-Impuls Sequenz (DS-Burst) und 12Impuls Sequenz (UWB-Burst) zeigen.

\subsection{Bewertung der Verfahren}

Für den in Abb. 6 dargestellten Steuerrechner ist lediglich das Ausgangssignal des HF-Frontends von Interesse. Daher wurden zum Vergleich beider Verfahren ausschließlich die mittels der NF-Ausgangssignale des AD-Wandlers erzielten TDoA-Ergebnisse betrachtet. 

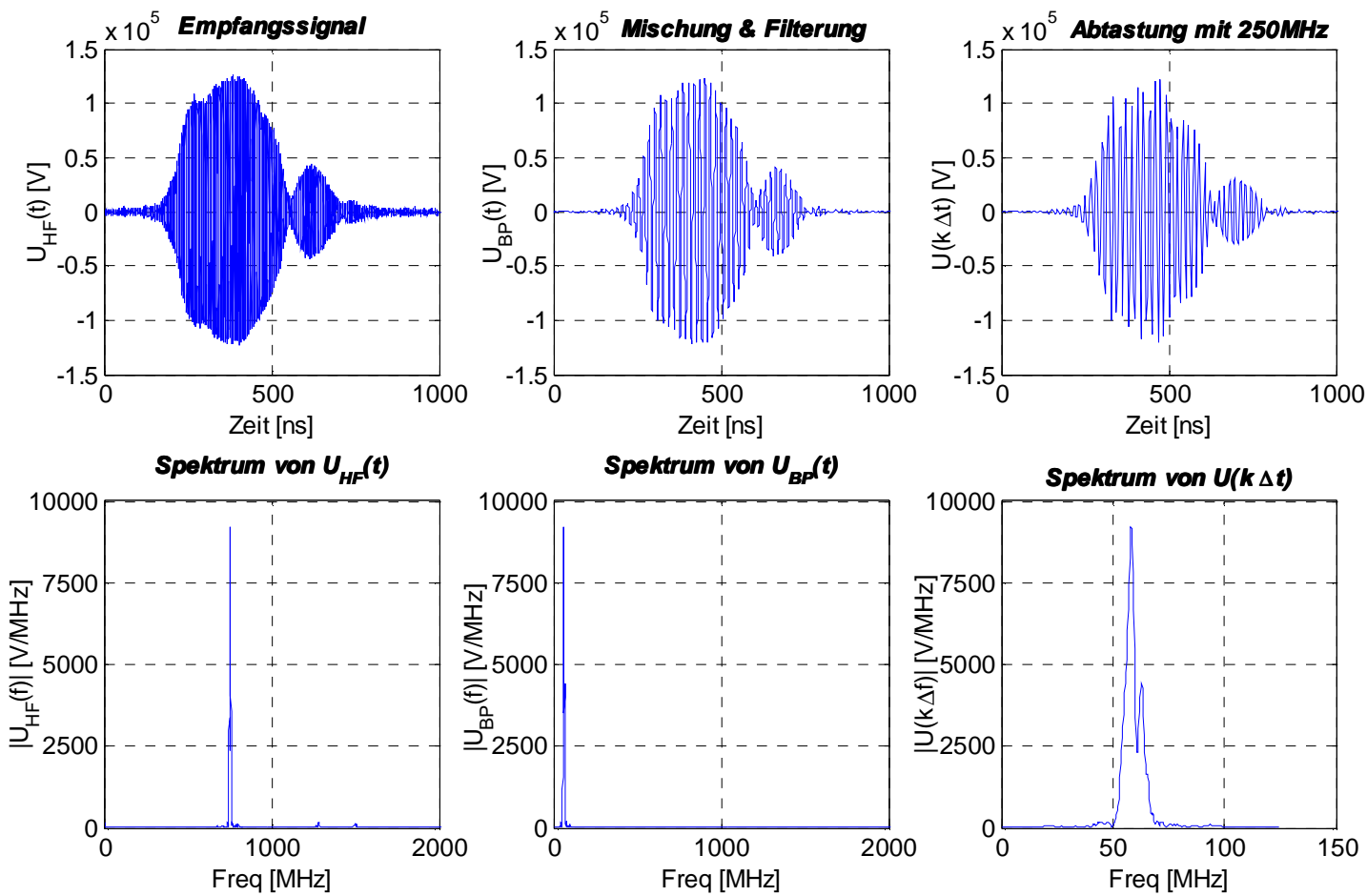

Abb. 7. Modell eines HPM-Pulses, welches von einer Empfangsstation detektiert und verarbeitet wurde: Zeitsignale (oben); zugehörige Spektren (unten).
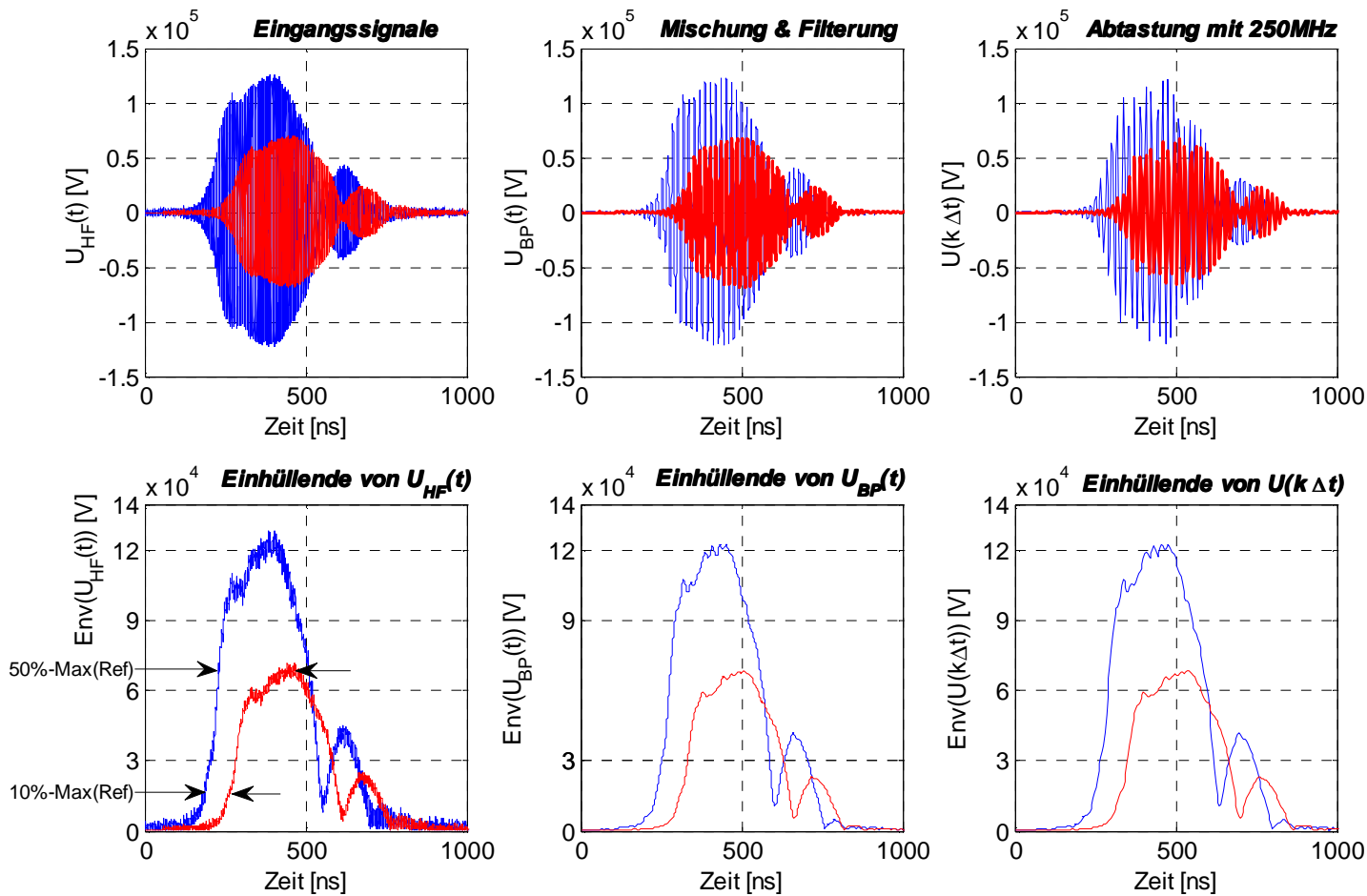

Abb. 8. Beispiel zur Erläuterung der Zeitungenauigkeit bei der Bestimmung der Signallaufzeitdifferenz ohne Korrekturmaßnahmen: $f_{\mathrm{LO}}=$ $690 \mathrm{MHz}$. 

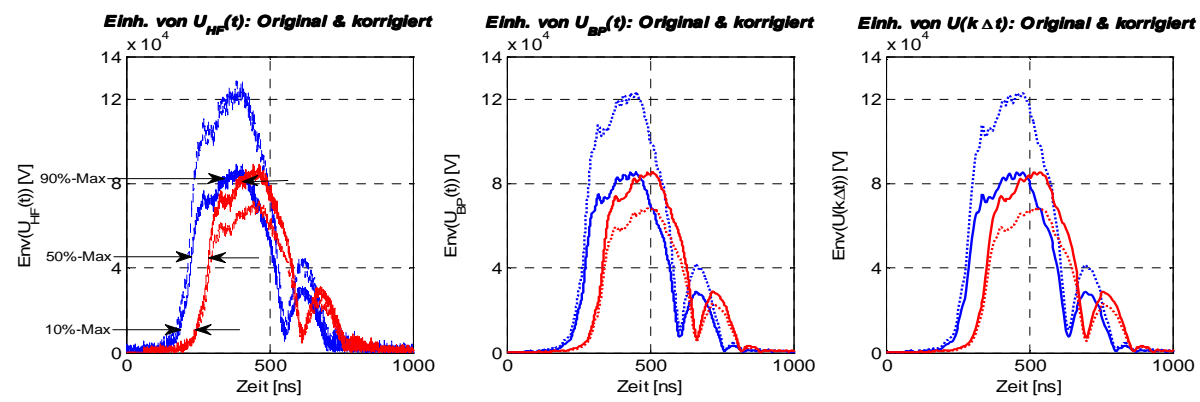

Abb. 9. Korrektur der Hüllkurven zwecks genauer Bestimmung der Laufzeitdifferenz: Gestrichelt (ohne Korrektur), durchgezogen (mit Korrektur).

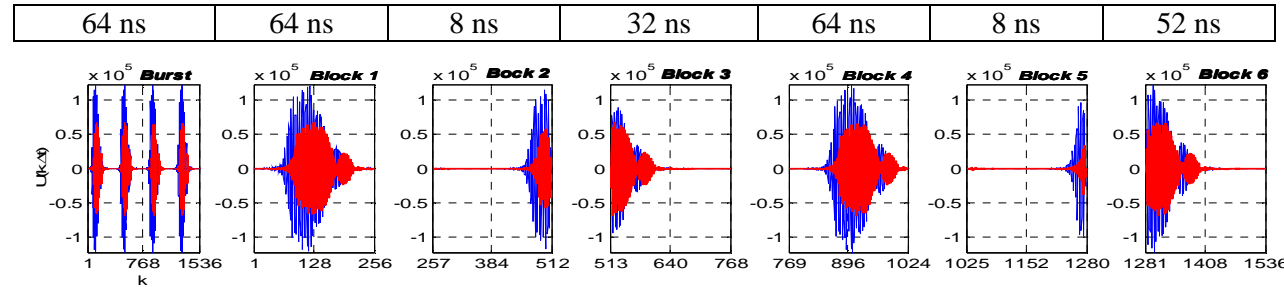

Abb. 10. Zerlegung der NF-Teilsignale eines HPM-Bursts für die Ermittlung der erforderlichen Signaldauer bei der Bestimmung der Laufzeitdifferenz mittels Korrelation: $f_{\mathrm{LO}}=690 \mathrm{MHz}$.

\begin{tabular}{|c|c|c|c|c|c|c|}
\hline $60 \mathrm{~ns}$ & $60 \mathrm{~ns}$ & $60 \mathrm{~ns}$ & $60 \mathrm{~ns}$ & $60 \mathrm{~ns}$ & $60 \mathrm{~ns}$ & $60 \mathrm{~ns}$ \\
\hline \\
010
\end{tabular}

Abb. 11. Segmentierung der NF-Teilsignale eines DS-Bursts in Blöcken von 128 Samples für die Ermittlung der Signaldauer bei der Berechnung der TDoA durch Korrelation: $f_{\mathrm{LO}}=290 \mathrm{MHz}$.

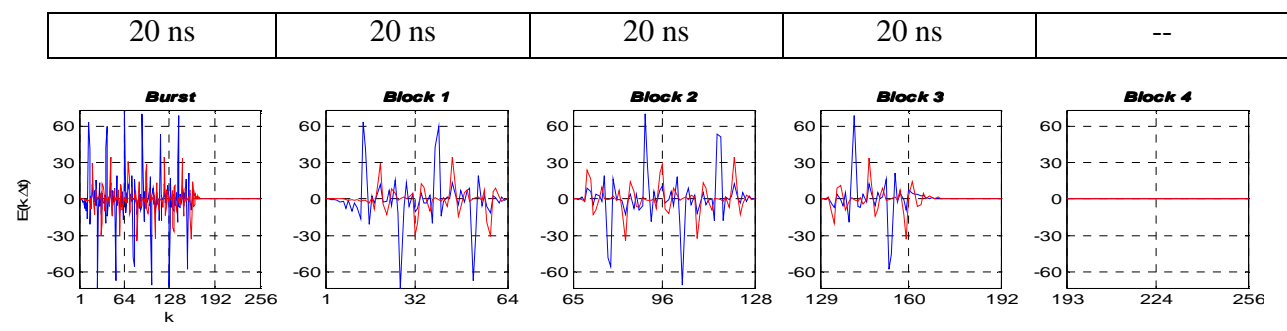

Abb. 12. Zerlegung der NF-Teilsignale eines UWB-Bursts in Blöcken von 64 Samples für die Ermittlung der Signaldauer bei der Berechnung der TDoA durch Korrelation: $f_{\mathrm{LO}}=290 \mathrm{MHz}$. 
Mit der Erkenntnis, dass die Verarbeitungskette des HFFrontends die Messergebnisse beeinflusst, ist die direkte Ermittlung der TDoA-Werte mittels Ereigniszeitpunkte mehr für HPM- und DS-Pulsformen geeignet (Vergleich $\left.\mathrm{U}_{\mathrm{HF}}(\mathrm{t})\right)_{\mid k o r}, \mathrm{U}_{\mathrm{BP}}(\mathrm{t}){ }_{\mid k \text { kor }}$ und $\mathrm{U}(\mathrm{k} \Delta \mathrm{t})_{\mid k \text { kor }}$ von Tabelle 2). Die Bestimmung der TDoA-Werte anhand Signalkorrelation hingegen ist für alle drei HPEM-Pulsformen gültig. Wichtig hierbei ist das Vorhandensein von genügend Bandbreite nach dem Mischvorgang, denn eine sinnvolle Signalkorrelation benötigt Bandbreite. Damit diese nötige Bandbreite zur Verfügung gestellt wird, sollte die Frequenz des Lokaloszillators im Frequenzbereich des Signalspektrums eingestellt werden, wo noch relevante Signalfrequenzen (z.B. in der Nähe der Mittenfrequenz) vorhanden sind.

\section{Zusammenfassung}

Die durchgeführten Untersuchungen haben gezeigt, dass die Berechnung der Laufzeitdifferenzen von mittels HPEMQuellen erzeugten Signalen sowohl durch Signalkorrelation als auch durch Festlegung eines Identifizierers möglich ist. Die Gültigkeit beider Verfahren - unabhängig von der eingesetzten Pulsform - ist nur für den Fall realisierbar, dass die Verarbeitungskette des HF-Frontends die Messdaten geringfügig beeinflusst (siehe Fall 20 ns Dalay in Tabelle 3).
Danksagung. Diese Arbeiten wurden durchgeführt im Rahmen des Verbundprojektes "Elektromagnetischer Schutz von Verkehrsinfrastrukturen (EMSIN)", das vom BMBF (Bundesministerium für Bildung und Forschung) unter dem Förderkennzeichen 13N10405 gefördert wird. Die Autoren bedanken sich bei Frau K. Reichel vom VDI Technologiezentrum GmbH für ihre Unterstützung.

\section{Literatur}

Bronstein, I. N., Semendjajew, K. A., Musiol, G., and Mühlig, H.: Taschenbuch der Mathematik, Verlag Harri Deutsch, Frankfurt am Main, 4., Überarbeitete und erweiterte Auflage der Neubearbeitung, ISBN 3-8171-2004-4, 1999.

Giri, D. V.: High-Power Electromagnetic Radiators: Nonlethal Weapons and Other Application, Harvard University Press, Cambridge, Massachusetts, and London, ISBN 0-674-01569-X, 2004.

Taylor, C. D., and Giri, D. V.: High-Power Microwave Systems and Effects, Taylor \& Francis, Washington, Bristol, and London, ISBN 1-674-56032-302-7, 1994.

Schmidt, H. U.: Technologische Aspekte asymmetrischer Bedrohungen, Bericht der Expertengruppe 4: Bedrohung durch elektromagnetische Effekte, November, 2002.

IEC 61000-2-13: 2005-03: Electromagnetic compatibility (EMC)Part 2-13: Environment - High-power electromagnetic (HPEM) environments, radiated and conducted, 2005.

Nguete Tsamo, R.: Erweiterung eines Ortungssystems und Verifikation der Leistungsfähigkeit, Masterarbeit bei Thales Defence \& Security Systems GmbH, Pforzheim, Deutschland, 2008.

Gutmann, F.: Positionsbestimmung in GSM- und UMTSNetzwerken, Seminararbeit beim Lehrstuhl für Kommunikationssyteme von der Albert Ludwigs Universität Freiburg, Juni, 2007. 